\title{
Temperature-dependent residual broadening of x-ray diffraction spectra in nanocrystalline plasticity
}

\author{
S. Brandstetter, Ž. Budrović, S. Van Petegem, B. Schmitt, E. Stergar, \\ P. M. Derlet, and H. Van Swygenhoven ${ }^{\text {a) }}$ \\ Paul Scherrer Institute, CH-5232 PSI-Villigen, Switzerland
}

(Received 16 May 2005; accepted 13 October 2005; published online 30 November 2005)

\begin{abstract}
In situ x-ray diffraction peak profile analysis at room temperature has shown that peak broadening during plastic deformation is reversible upon unloading for nanocrystalline metals, demonstrating the lack of a developing permanent dislocation network. In this letter, we show that the peak broadening is not reversible when similar load-unload cycles are performed at $180 \mathrm{~K}$. However, by then warming the sample to $300 \mathrm{~K}$, peak broadening recovers to a great extent and all subsequent plastic deformation load/unload cycles are characterized again by a reversible peak broadening. The temperature-dependent residual peak broadening provides explicit evidence of a thermal component in the nanocrystalline deformation mechanism. (C) 2005 American Institute of Physics.
\end{abstract}

[DOI: $10.1063 / 1.2138359$ ]

A number of key experiments on plastic deformation of nanocrystalline (nc) metals have been interpreted in terms of a dislocation picture suggested by atomistic simulations, where dislocations are emitted from grain boundaries (GBs), propagate and eventually are absorbed in neighboring GBs. ${ }^{1,2}$ Transition electron micropscopy (TEM) evidences dislocation activity ${ }^{3,4}$ but these types of analyses cannot reveal the dominant deformation mechanisms nor evidence GB accommodation processes. Stress relaxation or strain rate jump test experiments reveal a much lower activation volume and much higher value for the strain rate sensitivity than those measured for their coarse-grained counter parts ${ }^{5}$ and a model has been proposed to associate these volumes with the nucleation of dislocation loops from GBs. ${ }^{6} \mathrm{X}$-ray diffraction (XRD) technique probes predominantly the strain signature interior to the grains without strong interference from intrinsic GBs (Ref. 7) —an important feature when considering nanocrystalline systems. Indeed, room temperature in situ XRD experiments reveal that the peak broadening that occurs during plastic deformation is entirely reversible upon unloading for $\mathrm{nc}-\mathrm{Ni}$ with a mean grain size of $30 \mathrm{~nm}$ and a relatively narrow grain size distribution. ${ }^{8}$ This result demonstrates the absence of the formation of a permanent dislocation network within the grains during plastic deformation and suggests that peak broadening during loading comes from different stress signatures in different grains and/or from dislocations emitted from GBs, which are absorbed in GBs upon unloading. Reference 5 has revealed that by reducing temperatures down to the cryogenic regime, nanocrystalline metals can increase their yield strength by up to $80 \%$. Here we report on in situ XRD profile analysis at low temperature with the aim to reveal further the importance and nature of the thermal component in the nc deformation mechanism.

The material of choice is nc electrodeposited $\mathrm{Ni}$ with a mean grain size of 20-30 nm, using the same batch as for the experiments performed at room temperature. ${ }^{8}$ In situ tensile testing was performed at $180 \mathrm{~K}$ at the materials beamline of the Swiss Light Source, using $\mathrm{x}$ rays with an energy of 17.5

\footnotetext{
${ }^{a)}$ Electronic mail: helena.vs@psi.ch
}

$\mathrm{keV}$ and a multistrip detector allowing measurement of a 60 deg diffraction pattern at once. The sample was cooled with liquid nitrogen in a two chamber system using Kapton foils flushed with nitrogen gas to prevent ice formation. Two different types of loading histories are studied.

Figure 1(a) shows the stress-strain curves for five in situ loading-unloading cycles performed on nc-Ni at $180 \mathrm{~K}$ (colored lines) and for comparison, a similar curve for three loading-unloading cycles at room temperature is shown (dotted lines) taken from Ref. 8 . The behavior at $180 \mathrm{~K}$ differs
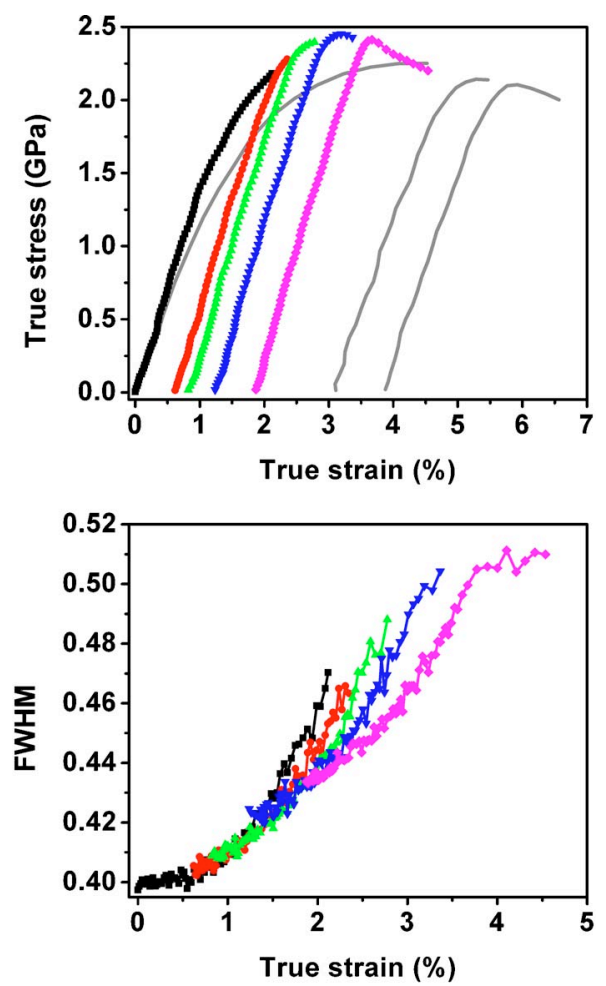

FIG. 1. (a) (Color online) The stress/strain curve performed at low temperature where the different loading runs are colored in black, red, green, blue, and purple. For comparison similar stress/strain curves for three loading runs at $300 \mathrm{~K}$ are displayed as shaded gray lines (taken from Ref. 8). (b) Peak width measured as FWHM of the (311) peak as a function of strain for five loading cycles. 


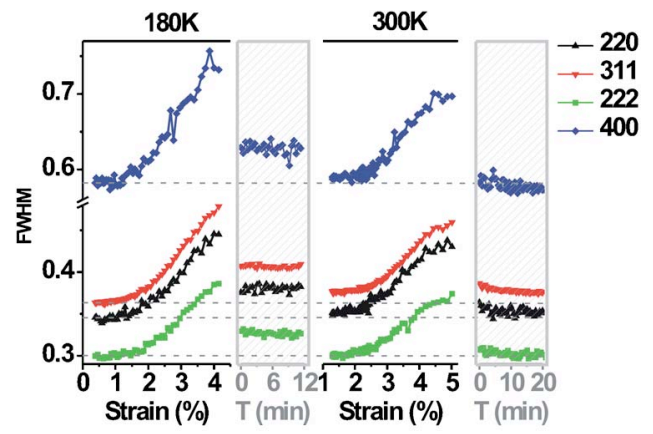

FIG. 2. (Color online) FWHM for a sample loaded and unloaded at $180 \mathrm{~K}$ and then $300 \mathrm{~K}$. Left two panels are the FWHM at $180 \mathrm{~K}$ during plastic loading as a function of strain and after unloading as a function of time. Right two panels are the FWHM at $300 \mathrm{~K}$ during plastic loading as a function of strain and after unloading as a function of time.

from that at RT in terms of a higher strength, a more pronounced hardening and a reduction in total elongation, the latter, however, not always being the case. In fact, out-ofbeam loading/unloading experiments did not exhibit a systematic difference in total elongation as a function of temperature indicating that fluctuations in elongation seem to be issued by sample flaws. In Fig. 1(b) the full width at half maximum (FWHM) of the (311) reflection is shown for the deformation at $180 \mathrm{~K}$, where the colors correspond to the colors of the loading/unloading cycles used in Fig. 1(a). Figure 1 (b) reveals that upon unloading at $180 \mathrm{~K}$ a fraction of the FWHM does not recover. This trend is seen for all diffraction peaks and is fundamentally different from the behavior of the peak broadening at RT, which was fully recoverable upon each unloading cycle. ${ }^{8}$

The earlier results calls for an additional test in which a sample, deformed and unloaded at $180 \mathrm{~K}$, is (in situ) warmed up to RT followed by an additional plastic deformation cycle at RT. Figure 2 shows the FWHM of the (220), (311), (222) and (400) diffraction peaks for this loading history-it is worth noting that during the initial cooling down to $180 \mathrm{~K}$ (prior to loading) peak widths did not change, whereas peak positions did according to thermal lattice contraction. The first part of this curve corresponds to the change in FWHM according to continuous deformation at $180 \mathrm{~K}$ until $4 \%$ total strain, followed by unloading at $180 \mathrm{~K}$. The second part of the curve corresponds to the FWHM during the loadingunloading cycle on the same sample now at RT. The first part of this figure demonstrates once again that upon unloading at $180 \mathrm{~K}$ the peak broadening is irreversible, however, what is striking is that all of this additional broadening recovers after warming up to RT with the exception to the trend being the (311) reflection, where $11 \%$ of the total broadening does not recover after warming up. When the sample is then additionally plastically deformed at RT, the FWHM is entirely reversible relative to the condition before room temperature deformation. It is worth to note that compared to the $180 \mathrm{~K}$ measurements, there is a time dependent component in the recovery of the FWHM. No changes in microstructure could be observed in TEM after deformation, which is in agreement with the observation that after loading-unloading at 180 $\mathrm{K}$, the FWHM for the majority of peaks recover to their original values when the sample is warmed up to room temperature.

The temperature dependent residual peak broadening demonstrates (1) that the mechanism responsible for plastic deformation in electrodeposited $\mathrm{nc}-\mathrm{Ni}$ is thermally activated and (2) that it is such that it leaves additional inhomogeneous strains in grain interiors upon unloading at low temperatures, whereas no such footprint is left at room temperature. That this footprint can be significantly removed upon warming up to room temperature, with a return to reversibility upon subsequent loading and unloading, demonstrates a well defined plasticity mechanism that does not significantly change the nc microstructure. In terms of the dislocation picture suggested by atomistic simulations, the additional inhomogeneous strains that remain upon unloading at $180 \mathrm{~K}$ can be understood by the "freezing-in" of dislocations due to a restriction in the atomic activity within the surrounding GB. Indeed, simulations suggest that atomic shuffling and stress assisted free volume migration are necessary for the nucleation, propagation and absorption of dislocations within the nc environment. ${ }^{9-11}$ Such atomic scale activity constitutes the heart of the thermal activation process, and is further evidenced by the increased strength seen in the low temperature deformation curves where higher stresses are needed to nucleate and propagate dislocations. Upon warming up, such GB atomic activity becomes more apparent allowing dislocations to propagate through the grain and to be absorbed in the surrounding GB structure. Such a picture evidences that not only nucleation, but also propagation and absorption has a thermal component, as also suggested by recent activation volume measurements. ${ }^{5}$ This is, however, not fully taken into account in the model proposed in Ref. 6 where after nucleation dislocation motion is considered to be predominantly an athermal event. In summary, our in situ experiments demonstrate temperature-dependent behavior in XRD reversible peak broadening, providing strong evidence for the thermally activated dislocation mechanism during tensile deformation of bulk nc metals.

The authors acknowledge the financial support of the Swiss-FN (Grant Nos. 2100-065152 and 200020-103714/1.

${ }^{1}$ H. Van Swygenhoven. Science 296, 66 (2002).

${ }^{2}$ H. Van Swygenhoven, P. M. Derlet, and A. G. Frøseth, Nat. Mater. 3, 399 (2004).

${ }^{3}$ R. C. Hugo, H. Kung, J. R. Weertman, R. Mitra, J. A. Knapp, and D. M. Follstaedt, Acta Mater. 51, 1937 (2003).

${ }^{4}$ K. S. Kumar, S. Suresh, M. F. Chisholm, J. A. Horton, and P. Wang, Acta Mater. 51, 387 (2003).

${ }^{5}$ Y. M. Wang and E. Ma, Appl. Phys. Lett. 85, 2750 (2004).

${ }^{6}$ R. J. Asaro and S. Suresh, Acta Mater. 53, 3369 (2005).

${ }^{7}$ P. M. Derlet, S. Van Petegem, and H. Van Swygenhoven, Phys. Rev. B 71, 024114 (2005)

${ }^{8}$ Z. Budrovic, H. Van Swygenhoven, P. M. Derlet, S. Van Petegem, and B. Schmitt, Science 304, 273 (2004).

${ }^{9}$ H. Van Swygenhoven, P. M. Derlet, and A. Hasnaoui, Phys. Rev. B 66, 024101 (2002).

${ }^{10}$ P. M. Derlet, H. Van Swygenhoven, and A. Hasnaoui, Philos. Mag. 83 3569 (2003).

${ }^{11}$ A. G. Frøseth, P. M. Derlet, and H. Van Swygenhoven, Acta Mater. 52, 5863 (2004). 